\title{
Can we apply the same indication of endoscopic submucosal dissection for primary gastric cancer to remnant gastric cancer?
}

\author{
Yoon Young Choi · In Gyu Kwon • Sang Kil Lee • Hyun Ki Kim • \\ Ji Yeong An · Hyoung Il Kim • Jae Ho Cheong • Richard Thomas Mliwa • \\ Sung Kwan Shin $\cdot$ Yong Chan Lee $\cdot$ Woo Jin Hyung $\cdot$ Sung Hoon Noh
}

Received: 3 January 2013/Accepted: 21 April 2013/Published online: 22 May 2013

(c) The International Gastric Cancer Association and The Japanese Gastric Cancer Association 2013

\begin{abstract}
Background Currently, remnant gastric cancer (RGC) is uncommon compared with gastric stump cancer, but early detection of gastric cancer and improved postsurgical survival will lead to increased incidence of RGC. Therefore, the indication of endoscopic submucosal dissection (ESD) for RGC is now required, but there have been no reports about this because of the lack of information for RGC.

Methods A retrospective review was conducted on 105 patients who underwent completion total gastrectomy (CTG) and 5 patients who underwent ESD for RGC between January 1998 and December 2010 at Yonsei University Hospital.

Results Forty-one (39\%) of 105 patients were diagnosed with early RGC. Among these patients, 6 had an absolute
\end{abstract}

Y. Y. Choi and I. G. Kwon contributed equally to this work.

Y. Y. Choi - I. G. Kwon · J. Y. An · H. I. Kim - J. H. Cheong ·

R. T. Mliwa - W. J. Hyung · S. H. Noh $(\bowtie)$

Department of Surgery, Yonsei University Health System,

Yonsei University College of Medicine, 134 Shinchon-dong,

Seodaemun-ku, Seoul 120-752, Republic of Korea

e-mail: sunghoonn@yuhs.ac

S. K. Lee · S. K. Shin · Y. C. Lee

Department of Gastroenterology, Yonsei University Health

System, Yonsei University College of Medicine, Seoul,

Republic of Korea

\section{H. K. Kim}

Department of Pathology, Yonsei University Health System,

Yonsei University College of Medicine, Seoul,

Republic of Korea

R. T. Mliwa

Department of Surgery, Muhimbili University of Health

and Allied Sciences, Dar es Salaam, Tanzania indication for ESD, whereas 11 met expanded criteria for ESD. In these patients, there was no association between the severity of the former gastric cancer and the current RGC. Also, none of these 17 patients had LN metastasis after CTG, and only $1(2.4 \%)$ of 41 early RGC patients had LN metastasis. Median operative time was $216 \mathrm{~min}$ for CTG and median hospital stay was 8 days. There were two major and five minor complications. One splenectomy was performed because of injury that occurred during CTG.

Conclusions Applying the indication of ESD for primary gastric cancer to RGC would be possible, and it could be an alternative treatment option for selected patients with RGC.

Keywords Remnant gastric cancer - Endoscopy - ESD . Lymph node - Gastric cancer

\section{Introduction}

Early gastric cancer (EGC) is highly curable with endoscopic resection or surgery. Treatment of selected EGC cases using endoscopy was popularized in the late 1980s with the development of endoscopic mucosal resection (EMR) [1]. Endoscopic submucosal dissection (ESD) now permits complete resection for more larger and deeper tumors without piecemeal resection [2]. Consequently, ESD has extended the indications for endoscopic tumor resection in the absence of evidence for lymph node (LN) metastasis [3-5].

In contrast to endoscopic procedures, radical surgery permits evaluation of LN metastasis and has previously been considered to be the only method for achieving cure of remnant gastric cancer (RGC). However, adhesions and anatomic deformation resulting from a previous 
gastrectomy with $\mathrm{LN}$ dissection increase the difficulty of the second operation, completion total gastrectomy (CTG) $[6,7]$. In the absence of LN metastasis, ESD offers the possibility of cure without the risks associated with a second oncological surgical procedure. Even though ESD has already been used to treat carcinoma in the remnant stomach [8-10], to date, there has been no decided indication of ESD for RGC because it had been uncommon and, consequently, there has been no report that reviewed a large amount of pathological results for RGC after CTG.

Because early detection of gastric cancer and improved postsurgical survival will lead to an increased incidence of RGC, we now need to decide the indication of ESD for RGC. If the former gastric cancer (FGC) did not affect the current RGC, applying the indication of ESD for primary gastric cancer to RGC is possible. To inquire into the relationship between FGC with current RGC and the possibility of using the same indication of ESD for RGC, we reviewed the surgical and pathological outcomes of patients who underwent CTG for RGC, especially those who were possible candidates for ESD.

\section{Patients and methods}

We performed a retrospective review of the data of patients who underwent CTG for RGC between January 1998 and December 2010 at Yonsei University Hospital. In the same period of time, there were 9,984 cases of conventional gastric cancer patients. This review was approved by the Institutional Review Board of our institution (4-20120639).

\section{Definitions}

RGC was defined by the following criteria: (1) a history of curative gastrectomy for gastric adenocarcinoma, (2) an interval from curative gastrectomy to identification of remnant gastric tumor of more than 12 months, and (3) pathologically confirmed adenocarcinoma in the remnant stomach. When the depth of tumor was limited to the mucosa or submucosa layer and the tumor was RGC, we defined it as early RGC. In relative concept, gastric stump cancer (GSC) was defined by the criteria of a previous gastrectomy for a benign condition, followed by diagnosis of cancer in the remnant stomach. Papillary adenocarcinoma as well as well or moderately differentiated tubular adenocarcinoma was classified as differentiated type. Poorly differentiated tubular adenocarcinoma, mucinous adenocarcinoma, and signet-ring cell carcinoma were classified as undifferentiated types.

We applied the same indication of ESD for primary gastric cancer to RGC. An absolute indication (AI) for ESD was defined by evidence of a non-ulcerative, differentiatedtype lesion, limited to the gastric mucosa and $<20 \mathrm{~mm}$ in maximal dimension. Expanded indication (EI) for ESD included (1) a non-ulcerative, differentiated tumor confined to the gastric mucosa irrespective of size, (2) an ulcerative, differentiated tumor confined to the gastric mucosa and $<30 \mathrm{~mm}$ in maximal dimension, (3) a non-ulcerative, undifferentiated tumor confined to the gastric mucosa and $<20 \mathrm{~mm}$ in maximal dimension, or (4) a differentiated tumor confined to the submucosa $(\mathrm{sm} 1 ;<0.5 \mathrm{~mm}$ from the muscularis mucosa) and $<30 \mathrm{~mm}$ in maximal dimension [11].

Investigated outcomes

We reviewed the characteristics of FGC, including the former tumor stage, type of surgical reconstruction, and interval between previous gastrectomy and CTG. We also investigated the characteristics of RGC, such as tumor location, tumor size, presence of ulcers, tumor histology, $\mathrm{LN}$ metastasis, and pathological T-stage. The operative time and in-patient hospitalization, treatment complications, patient survival, and disease recurrence following CTG were reviewed to inquire the postoperative outcomes. Procedural complications were divided into major and minor categories according to the necessity for interventional procedures.

\section{Results}

Characteristics of patients who underwent CTG for early RGC

In total, 105 patients underwent CTG for RGC, and among them, 41 (39\%) had early RGC. Six (14.6\%) of the 41 patients met the AI criteria for ESD, whereas $11(26.8 \%)$ met the EI criteria for ESD (Fig. 1). The median age of 17 patients who were possible candidates for ESD was 66 years (range 33-76 years), and 5 patients $(29.4 \%)$ were female. More than two-thirds $(70.6 \%)$ of patients had undergone previous Billroth II reconstruction procedures. Because 5 of the 17 patients who met ESD criteria underwent previous gastrectomy at other hospitals, the exact extent of previous LN dissection could not be determined. The stage of FGC varied from T1N0 to T4N1a. However, the exact tumor stage was unknown in 3 patients. The median interval from previous gastrectomy to CTG was 46 months (range 13-365 months; Table 1).

Among these possible candidates for ESD, RGC occurred at the anastomosis site in 7 patients $(41.2 \%)$. Six of these patients underwent Billroth II reconstruction (Tables 1, 2). There was no evidence of $\mathrm{LN}$ metastasis 
Fig. 1 Characteristics of remnant gastric cancer $(R G C)$. $E S D$ endoscopic submucosal dissection

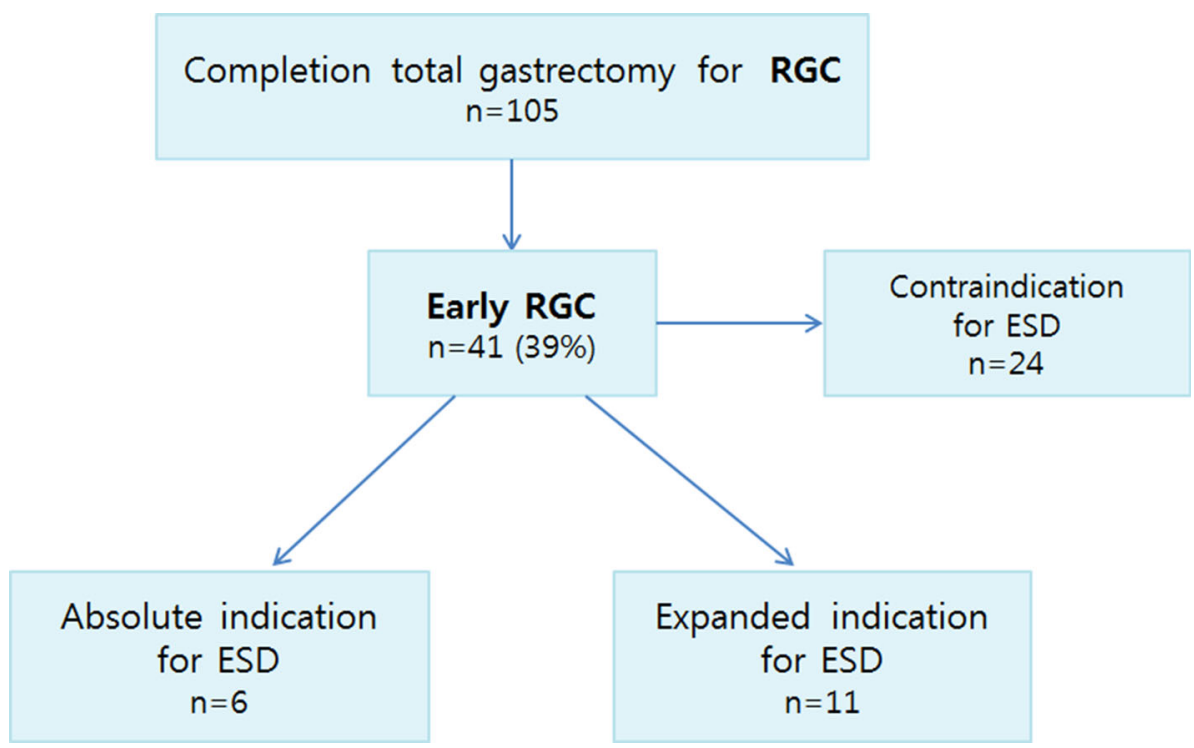

Table 1 Characteristics of former gastric cancer patients who met either an absolute indication or expanded indication for endoscopic submucosal dissection but underwent completion total gastrectomy (CTG)
$L N$ lymph node, $C T G$

completion total gastrectomy,

$D G$ distal gastrectomy,

BII Billroth II, BI Billroth I,

$E S D$ endoscopic submucosal dissection, $A I$ absolute indication, $E I$ expanded indication, $M$ male, $F$ female

a Age at the time of CTG (years)

b Failed to acquire information about pathological reports of previous operation

\begin{tabular}{llllllc}
\hline $\begin{array}{l}\text { Number of } \\
\text { case }\end{array}$ & Age $^{\mathrm{a}}$ & Sex & $\begin{array}{l}\text { Reconstruction } \\
\text { type }\end{array}$ & $\begin{array}{l}\text { LN } \\
\text { dissection }\end{array}$ & Stage & $\begin{array}{l}\text { Interval to } \\
\text { CTG (months) }\end{array}$ \\
\hline $\begin{array}{lllll}\text { AI for ESD } \\
1\end{array}$ & & & & & T1N0 & 17 \\
2 & 45 & M & DG with BII & D2 & T3N1 & 33 \\
3 & 71 & M & DG with BII & D2 & $-{ }^{\text {b }}$ & 332 \\
4 & 66 & M & DG with BII & - & $-{ }^{\text {b }}$ & 341 \\
5 & 73 & M & DG with BII & - & T2N0 & 37 \\
6 & 76 & M & DG with BII & D1+ & T1N0 & 20 \\
EI for ESD & 66 & M & DG with BII & - & & \\
7 & 70 & M & DG with BII & D2 & T3N1 & 59 \\
8 & 52 & M & DG with BII & D2 & T1N0 & 112 \\
9 & 40 & M & DG with BI & D2 & T1N0 & 25 \\
10 & 68 & M & DG with BII & - & $-{ }^{\mathrm{b}}$ & 365 \\
11 & 47 & F & DG with BII & D1+ & T1N0 & 13 \\
12 & 74 & M & DG with BII & D2 & T3N0 & 26 \\
13 & 63 & M & DG with BI & D1+ & T1N0 & 113 \\
14 & 33 & F & DG with BI & D2 & T2N2 & 34 \\
15 & 59 & F & DG with BI & - & T1N0 & 46 \\
16 & 43 & F & DG with BII & D1+ & T4N1 & 76 \\
17 & 66 & F & DG with BI & D2 & T1N0 & 50 \\
\hline & & & & & \\
\hline
\end{tabular}

after CTG for RGC among these 17 patients. The duration of follow-up from CTG ranged from 18 to 98 months. One patient died of primary lung cancer, and follow-up was censored in 2 patients. Among the 41 patients diagnosed with early RGC, only 1 patient $(2.4,0-7.1 \%)$ showed evidence of metastasis in 1 of 10 harvested LNs. The tumor in this patient invaded the submucosa $(\mathrm{sm} 2,>0.5 \mathrm{~mm}$ from the muscularis mucosa); it measured $2.5 \mathrm{~cm}$ in length and was classified as a well-differentiated tubular adenocarcinoma (Table 3).
Postoperative outcomes after CTG for RGC

Table 4 shows the postoperative outcomes of CTG for RGC. The median operative time was $216 \mathrm{~min}$, and the median hospital stay was 8 days. One patient underwent combined splenectomy during CTG because adhesiolysis caused injury to the spleen, and five patients experienced minor complications after CTG for RGC: three patients developed atelectasis, one developed anemia requiring transfusion with packed red blood cells, and one developed 
Table 2 Characteristics of patients who met either an absolute indication or expanded indication for endoscopic submucosal dissection but underwent completion total gastrectomy

\begin{tabular}{|c|c|c|c|c|c|c|c|c|c|}
\hline $\begin{array}{l}\text { Number of } \\
\text { case }\end{array}$ & $\mathrm{Age}^{\mathrm{b}}$ & Sex & T-stage & $\begin{array}{l}\text { Histological } \\
\text { type }\end{array}$ & $\begin{array}{l}\text { Presence } \\
\text { of ulcer }\end{array}$ & $\begin{array}{l}\text { Size } \\
(\mathrm{mm})\end{array}$ & Location & $\begin{array}{l}\text { Lymph Node } \\
\text { (positive LN/ } \\
\text { retrieved LN) }\end{array}$ & $\begin{array}{l}\text { Duration of } \\
\text { follow up } \\
\text { (months) }\end{array}$ \\
\hline \multicolumn{10}{|l|}{ AI for ESD } \\
\hline 1 & 45 & M & $\mathrm{m}$ & Diff & - & $\leq 20$ & NAS & $0 / 6$ & 145 \\
\hline 2 & 71 & M & $\mathrm{m}$ & Diff & - & $\leq 20$ & NAS & $0 / 11$ & $79^{\mathrm{a}}$ \\
\hline 3 & 66 & M & $\mathrm{m}$ & Diff & - & $\leq 20$ & Anastomosis site & $0 / 19$ & 81 \\
\hline 4 & 73 & M & $\mathrm{m}$ & Diff & - & $\leq 20$ & Anastomosis site & $0 / 13$ & 24 \\
\hline 5 & 76 & M & $\mathrm{m}$ & Diff & - & $\leq 20$ & Anastomosis site & $0 / 8$ & 30 \\
\hline 6 & 66 & M & $\mathrm{m}$ & Diff & - & $\leq 20$ & NAS & $0 / 1$ & 24 \\
\hline \multicolumn{10}{|l|}{ EI for ESD } \\
\hline 7 & 70 & M & $\mathrm{m}$ & Undiff & - & $\leq 20$ & Anastomosis site & $0 / 10$ & $98^{\mathrm{c}}$ \\
\hline 8 & 52 & M & $\mathrm{m}$ & Undiff & - & $\leq 20$ & NAS & $0 / 0$ & 73 \\
\hline 9 & 40 & M & $\mathrm{m}$ & Undiff & - & $\leq 20$ & NAS & $0 / 0$ & 18 \\
\hline 10 & 68 & M & $\mathrm{m}$ & Diff & - & $\leq 30$ & Anastomosis site & $0 / 52$ & $75^{\mathrm{a}}$ \\
\hline 11 & 47 & $\mathrm{~F}$ & $\mathrm{~m}$ & Undiff & - & $\leq 20$ & NAS & $0 / 2$ & 18 \\
\hline 12 & 74 & M & $\mathrm{m}$ & Undiff & - & $\leq 20$ & Anastomosis site & $0 / 5$ & 41 \\
\hline 13 & 63 & M & $\mathrm{m}$ & Undiff & - & $\leq 20$ & Anastomosis site & $0 / 11$ & 35 \\
\hline 14 & 33 & $\mathrm{~F}$ & $\mathrm{~m}$ & Undiff & - & $\leq 20$ & NAS & $0 / 15$ & 44 \\
\hline 15 & 59 & $\mathrm{~F}$ & $\mathrm{~m}$ & Undiff & - & $\leq 20$ & NAS & $0 / 8$ & 24 \\
\hline 16 & 43 & $\mathrm{~F}$ & $\mathrm{~m}$ & Undiff & - & $\leq 20$ & NAS & $0 / 3$ & 18 \\
\hline 17 & 66 & $\mathrm{~F}$ & sm1 & Diff & & $\leq 30$ & NAS & $0 / 1$ & 30 \\
\hline
\end{tabular}

$N A S$ non-anastomosis site, $L N$ lymph node, $A I$ absolute indication, $E I$ expanded indication, $E S D$ endoscopic submucosal dissection, $M$ male, $F$ female, $m$ mucosa, $s m$ submucosa, Diff differentiated type, Undiff undifferentiated type

${ }^{\text {a }}$ Lost to follow-up at the indicated time

b Age at the time of completion total gastrectomy (years)

c The patient died secondary to sepsis following lung wedge resection for primary lung cancer

Table 3 Lymph node characteristics in patients who underwent completion total gastrectomy for early remnant gastric cancer

\begin{tabular}{clll}
\hline & pT-stage & $\begin{array}{l}\text { Number of patients } \\
\text { (LN + patients/total } \\
\text { patients) }\end{array}$ & $\begin{array}{l}\text { LN (positive } \\
\text { LN/retrieved } \\
\text { LN) }\end{array}$ \\
\hline $\begin{array}{clll}\text { Early RGC } \\
(n=41)\end{array}$ & $\mathrm{m}$ & $0 / 25$ & $0 / 224$ \\
& $\mathrm{sm}$ & $1 \mathrm{a} / 16(6.3 \%, 0-18.2 \%)$ & $1 / 120$ \\
& Total & $1 / 41(2.4 \%, 0-7.1 \%)$ & $1 / 344$ \\
\hline
\end{tabular}

$L N$ lymph node, $R G C$ remnant gastric cancer, $m$ mucosa, $s m$ submucosa

${ }^{a}$ A $2.5 \times 2 \mathrm{~cm}$ tumor invading the submucosa $(\mathrm{sm} 2,>0.5 \mathrm{~mm}$ from the muscularis mucosa): classified histologically as a well-differentiated tubular adenocarcinoma

an intraabdominal abscess, which required a change in intravenous antibiotics. One patient developed a pleural effusion and an intraabdominal abscess, which required external percutaneous drainage. One patient required reoperation because of leakage from the duodenal stump with A-loop obstruction and subsequently required admission to the intensive care unit.

\section{Discussion}

CTG for GSC is technically more challenging than total gastrectomy for primary gastric cancer because of the postoperative adhesions. However, because of previous extensive LN dissection, RGC is usually associated with more severe adhesions around the remnant stomach than GSC. Our results represented the difficulty of CTG for RGC: we identified five cases of minor complications and two cases of major complications, including reoperation, after CTG for RGC. Also, there was one combined splenectomy with CTG because of injury during the operation. Therefore, ESD prevents unnecessary complications and may be a better option if it applied with reasonable indication.

The present results showed that the severity of FGC was not associated with the interval to CTG or with survival after CTG for RGC. We also found no evidence of LN metastasis in patients who were candidates for ESD. In fact, the incidence of LN metastasis in early RGC was only $2.4 \%(0-7.1 \%)$ in our data, which is similar to the reported incidence of LN metastasis of $8.9 \%$ (467 of 5,265 
Table 4 Postoperative outcomes of completion total gastrectomy for remnant gastric cancer

\begin{tabular}{ll}
\hline & CTG $(n=17$, range), [NAS $(n=10)]$ \\
\hline OP time (min) & $216(125-300),[218(145-290)]$ \\
Hospital stay (days) & $8(6-83),[9(6-83)]$ \\
Complications & Minor \\
& Atelectasis: 3 (1 NAS, 2 anastomosis) \\
& Transfusion: 1 (NAS) ${ }^{\mathrm{a}}$ \\
& Intraabdominal abscess: 1 (NAS) \\
& Major \\
& Intraabdominal abscess with \\
& pleural effusion: 1 (NAS) \\
& Reoperation with intensive care \\
& unit care: 1 (NAS) \\
& Other \\
& Combined splenectomy resulting from injury
\end{tabular}

NAS non-anastomosis site, CTG completion total gastrectomy, OP operative

${ }^{a}$ Both complications occurred in one patient

patients) in primary early gastric cancer [3]. This similarity between RGC and primary gastric cancer suggests that the LN metastasis of RGC is affected by current stage and not by the stage of the FGC. Previous studies that reported the prognosis of patients with RGC showed similar results. One report did not find evidence of $\mathrm{LN}$ metastasis in 9 patients with early RGC [12], and RGC itself was not associated with poor survival [13]; another report failed to identify a statistically significant difference in survival between RGC and GSC [14]. One study reported that the survival of patients with pT1 stage RGC was $76 \%$ [15], a result worse than those of recent reports of survival from primary early gastric cancer, but this finding was based on data collected in the 1970s. Therefore, applying the indication of ESD for primary gastric cancer to RGC would be possible.

Despite the reported success of ESD for RGC or GSC $[8,16,17]$, ESD is also technically challenging in the remnant stomach. Even when the patient has an entire stomach, difficulty with enfacement and access to the lesion can increase the frequency of hemorrhage during and after ESD if the lesion is located in the upper third of the stomach [18]. In the case of the remnant stomach, the narrow space and fibrotic change around the anastomosis site increase the difficulty of ESD.

Others have hypothesized that use of staples may complicate ESD when RGC arises on a stapled anastomosis site. There have been a few reports about ESD for gastric cancer on a stapler site: one case report described successful ESD of a gastric tube cancer that arose on a previous surgical staple line [19], and another suggested that en bloc ESD can be highly effective, despite the presence of staples, when performed by an experienced endoscopist [8]. These reports could support the technical possibility of ESD for RGC on a stapler site, but the technical feasibility of ESD for RGC on an anastomosis site, a stapler site, should be carefully considered. In addition, the accuracy of preoperative endoscopic ultrasound was reported as $75 \%$, and even for T1 stage was only $77 \%$ [20]. It would be more difficult to estimate the real depth of tumor invasion than primary gastric cancer if the tumor is located on the anastomosis site, especially for a staple site. Thus, the accuracy of preoperative diagnosis would decrease for tumors on the anastomosis site and might be even worse for a stapler site. For these reasons it would be better not to select ESD when the tumor is located on an anastomosis site, especially on a stapler site.

We believe that early detection and advances in treatment for primary gastric cancer suggest that the incidence of RGC will gradually increase. Prior studies have suggested a role for ESD in the setting of RGC or GSC [8, 16, 17]. However, to the best of our knowledge, ours is the first study to review the pathological results of RGC with the incidence of LN metastasis. In fact, the critical aspect of this study is the limited number of cases: there were only 17 cases of possible candidates of ESD for RGC. This limitation would be resolved by future individual patient data meta-analysis or prospective multicenter studies, which will offer additional information and concrete evidence regarding the indications of ESD for RGC.

\section{Conclusions}

The present results showed the possibility to apply the indications of ESD for primary gastric cancer to RGC. Thus, ESD could be an alternative treatment option for selected patients with RGC. A future multicenter study or systematic review is required to decide the indications of ESD for RGC with more concrete evidence.

Acknowledgments This study was supported by eVelos system, which is a web-based clinical study management system (NCC Grant, NCC 1210120-1). This study was supported by a grant of the Korea Healthcare Technology R\&D Project, Ministry of Health and Welfare, Republic of Korea (A102065).

Conflict of interest None.

\section{References}

1. Tada M, Murakami A, Karita M, Yanai H, Okita K. Endoscopic resection of early gastric cancer. Endoscopy. 1993;25:445-50.

2. Ono H, Kondo H, Gotoda T, Shirao K, Yamaguchi H, Saito D, et al. Endoscopic mucosal resection for treatment of early gastric cancer. Gut. 2001;48:225-9. 
3. Gotoda T. Incidence of lymph node metastasis from early gastric cancer: estimation with a large number of cases at two large centers. Gastric Cancer. 2000;3:219-25.

4. Soetikno R, Kaltenbach T, Yeh R, Gotoda T. Endoscopic mucosal resection for early cancers of the upper gastrointestinal tract. J Clin Oncol. 2005;23:4490-8.

5. Tanaka M, Ono H, Hasuike N, Takizawa K. Endoscopic submucosal dissection of early gastric cancer. Digestion. 2008;77(suppl 1):23-8.

6. Lissens P, Filez L, Aerts R, D'Hoore A, Van Cutsem E, Ectors N, et al. Surgery for gastric remnant carcinoma following Billroth II gastrectomy. Eur J Surg Oncol. 1997;23:518-21.

7. Ahn HS, Kim JW, Yoo M, Park DJ, Lee H, Lee KU, et al. Clinicopathological features and surgical outcomes of patients with remnant gastric cancer after a distal gastrectomy. Ann Surg Oncol. 2008;15:1632-9.

8. Takenaka R, Kawahara Y, Okada H, Tsuzuki T, Yagi S, Kato J, et al. Endoscopic submucosal dissection for cancers of the remnant stomach after distal gastrectomy. Gastrointest Endosc. 2008;67:359-63.

9. Lee JY, Choi IJ, Cho S, Kim CG, Kook M, Ryu KW, et al. Endoscopic submucosal dissection for metachronous tumor in the remnant stomach after distal gastrectomy. Surg Endosc. 2010;24:1360-6.

10. Nishide $\mathrm{N}$, Ono H, Kakushima N, Takizawa K, Tanaka M, Matsubayashi $\mathrm{H}$, et al. Clinical outcomes of endoscopic submucosal dissection for early gastric cancer in remnant stomach or gastric tube. Endoscopy. 2012;44:577-83.

11. Japanese Gastric Cancer Association. Japanese gastric cancer treatment guidelines 2010 (ver. 3). Gastric Cancer. 2011;14: $113-23$.
12. Takeda J, Toyonaga A, Koufuji K, Kodama I, Aoyagi K, Yano S, et al. Early gastric cancer in the remnant stomach. Hepatogastroenterology. 1998;45:1907-11.

13. Kidokoro T, Hayashida Y, Urabe M. Long-term surgical results of carcinoma of the gastric remnant: a statistical analysis of 613 patients from 98 institutions. World J Surg. 1985;9:966-71.

14. Furukawa H, Iwanaga $T$, Hiratsuka M, Imaoka S, Ishikawa $O$, Kabuto $\mathrm{T}$, et al. Gastric remnant cancer as a metachronous multiple lesion. Br J Surg. 1993;80:54-6.

15. Ohashi M, Katai H, Fukagawa T, Gotoda T, Sano T, Sasako M. Cancer of the gastric stump following distal gastrectomy for cancer. Br J Surg. 2007;94:92-5.

16. Hosokawa O, Kaizaki Y, Watanabe K, Hattori M, Douden K, Hayashi $\mathrm{H}$, et al. Endoscopic surveillance for gastric remnant cancer after early cancer surgery. Endoscopy. 2002;34:469-73.

17. Hirasaki S, Kanzaki H, Matsubara M, Fujita K, Matsumura S, Suzuki S. Treatment of gastric remnant cancer post distal gastrectomy by endoscopic submucosal dissection using an insulation-tipped diathermic knife. World J Gastroenterol. 2008;14: 2550-5.

18. Isomoto H, Shikuwa S, Yamaguchi N, Fukuda E, Ikeda K, Nishiyama $\mathrm{H}$, et al. Endoscopic submucosal dissection for early gastric cancer: a large-scale feasibility study. Gut. 2009;58:331-6.

19. Yoshida T, Fukahara T, Inoue A, Sakurazawa K, Sasabe M, Ito T. One-piece endoscopic resection of a gastric tube cancer on the surgical staple line. Gastrointest Endosc. 2006;63:188-90.

20. Cardoso R, Coburn N, Seevaratnam R, Sutradhar R, Lourenco LG, Mahar A, et al. A systematic review and meta-analysis of the utility of EUS for preoperative staging for gastric cancer. Gastric Cancer. 2012;15:S19-26. 